\title{
Kristin Prüssner
}

\section{Rheuma? Mit 13 Jahren?}

Als ich 13 war, brach für mich erst mal eine Welt zusammen: Ich wurde krank, hatte Schmerzen und fühlte mich wie ein Außenseiter. Mit einer rheumatoiden Arthritis zu leben, war nicht immer leicht, besonders in der Schulzeit. Anders als die Freundinnen $\mathrm{zu}$ sein, ist nie das, was man sich als Teenie vorstellt. Mein Glück: Ich kam schnell in eine Rheumaklinik mit Kindern und Jugendstation. Ärzte, Pflegepersonal und Therapeuten verstanden meine körperlichen Schmerzen, aber auch meine seelischen Sorgen und Ängste. Ich bekam unzählige hilfreiche Therapien wie Physiotherapie, Wassertherapie, einen Psychologen und eine Auswahl an Medikamenten. Ich lernte in vielen Schulungen, mit meiner Krankheit umzugehen. Außerdem war es für mich total hilfreich, dass ich mich mit Gleichaltrigen austauschen konnte. Endlich fühlte ich mich nicht mehr so alleine! Ich fühle mich bei allem unterstützt und handlungsfähig, denn ich habe gemeinsam mit den Ärzten und Therapeuten viele Möglichkeiten, um mein Rheuma nicht die Oberhand gewinnen zu lassen.

Doch irgendwann begann die Zeit, in der man sich nicht mehr nur um die Schule, sondern auch um seine berufliche Zukunft Gedanken macht. Schon früh war für mich klar, dass ich Notärztin im Rettungshubschrauber werden wollte. Nach einem Praktikum in einer Klinik begann ich eine Ausbildung zur Kinderkrankenschwester. Dies sollte eine Zwischenstation auf dem Weg zur Ärztin werden.

Im Hinterkopf hatte ich bei all meinen Plänen natürlich immer das Rheuma. Bei mir sind auch die Hände betroffen, was mich manchmal trotz allgemein guter Verfassung ziemlich außer Gefecht setzen kann. Am Anfang der Ausbildung war ich total in meinem Element, vor allem bei meinem ersten Einsatz auf der Frühchen-Intensivstation - so viele süße Babys, die ich versorgen durfte! Aber dann, nach wenigen Monaten Ausbildung, bekam ich einen Schub und konnte nicht einmal mehr die Babys tragen. In den Gesprächen mit meiner Praxisanleiterin stellte ich für mich fest: So will ich nicht arbeiten. Ich möchte nicht regelmäßig fehlen, „nur“ weil meine Hände schlappmachen.

Also beschloss ich schweren Herzens gemeinsam mit meiner Praxisanleiterin, dass es auf diesem Weg für mich nicht weitergeht. Doch stand ich nun auf einmal ohne Plan da - und war sauer auf meinen Körper. Und mein Rheuma! Trotzdem war mir klar: Mein Platz ist im Gesundheitswesen. Da ich meine Heimatstadt Bielefeld möglichst nicht verlassen wollte, erkundigte ich mich, was die Uni Bielefeld an Studiengängen zu bieten hat. Dabei stieß ich auf „Health Communication“. Für mich war erst einmal das Wichtigste, dass ich dafür nicht unbedingt

Ә OpenAccess. (C) 2021 Kristin Prüssner, publiziert von De Gruyter. (cc))BY-NC-SA Dieses Werk ist lizenziert unter einer Creative Commons Namensnennung - Nicht kommerziell - Weitergabe unter gleichen Bedingungen 4.0 International Lizenz. https://doi.org/10.1515/9783110713336-041 
mit meinen Händen, sondern hauptsächlich mit meinem Kopf arbeiten muss. Ich hatte mein Rheuma gewissermaßen ausgetrickst. Damit hatte ich ein neues Ziel und freute mich enorm auf meine neue Perspektive.

Nun habe ich meinen Studiengang bereits abgeschlossen, ein Praktikum in der Schweiz absolviert und anschließend dort einen Traumjob gefunden - mit einem sehr verständnisvollen Arbeitgeber. Ich habe beispielsweise jede Woche die Möglichkeit, im Home Office zu arbeiten. Dies nimmt mir vor allem morgens den Zeitdruck und ermöglicht es mir an weniger guten Tagen, trotz Einschränkungen etwas leisten zu können. Heute arbeite ich als Produkt- und Salesmanagerin bei einem Softwareanbieter für Qualitäts-Managementsysteme. Ich bin dem Thema Qualitätsmanagement treu geblieben, jedoch bin ich nun in allen Branchen unterwegs und nicht mehr speziell im Gesundheitssystem. Ohne die abgebrochene Ausbildung wäre ich vermutlich nie zu diesem Studiengang gekommen. Vor allem aber bereue ich nicht, dass ich vorher die Ausbildung - trotz Zweifeln - ausprobiert habe! Selbst in der kurzen Zeit habe ich viele schöne Erfahrungen gemacht und umso mehr über das Gesundheitswesen, aber auch mich selbst gelernt. Ich hätte mich ohne den Versuch immer gefragt, ob ich die Ausbildung nicht doch geschafft hätte. Wir mit unserem Handicap müssen lernen, dass es okay ist, wenn etwas nicht klappt.

Die Krankheit muss sich nach meinem Leben richten. Statt zu verzichten, muss ich mich höchstens fragen: Wenn ich mal wieder die Welt entdecken will, wie organisiere ich den Transport und die richtige Lagerung der Medikamente? Mit Kühlsystemen und Kühlschränken in Hotelzimmern habe ich auch genügend Möglichkeiten, sie über einen längeren Weg hinweg aufzubewahren oder im Ausland an notwendige Medikamente zu kommen. Trotz einiger Einschränkungen, die mir das Rheuma beschert, habe ich in der heutigen Zeit doch den Vorteil, dass mir die Behandlungen viele Freiheiten in der Lebensgestaltung erhalten. Mir steht regelmäßig meine Physiotherapie zur Seite, um schnell auf Beschwerden reagieren zu können und meine grundsätzliche körperliche Verfassung zu stärken. Mit meinem Rheumatologen habe ich eine Auswahl an Medikamenten zur Verfügung, um daraus die für mich beste Therapie zu wählen. Die meisten davon sind ohne schwerwiegende Nebenwirkungen und sehr wirksam. Für mich selbstverständlich - für ältere Patienten ganz und gar nicht.

\section{Chancen für die Zukunft}

Das bedeutet nicht nur für meine aktuelle Situation einen besseren Status quo, sondern auch für den Verlauf meiner Erkrankung und für meine Zukunft. Somit sehe ich mich mit meiner Erkrankung wohl immer noch als Glückskind. Rheuma 
hätte ich so oder so. Dank der heutigen Therapiemöglichkeiten habe ich aber ein fast normales Leben, vor allem, wenn ich mir überlege, was die Erkrankung für Menschen wie mich bedeutet hätte, bevor es wirksame Medikamente gab. Und wer weiß, vielleicht wird Rheuma ja tatsächlich eines Tages heilbar. 